\title{
Geoneutrinos in large direct detection experiments
}

\author{
Graciela B. Gelmini, ${ }^{1, *}$ Volodymyr Takhistov, ${ }^{1, \dagger}$ and Samuel J. Witte ${ }^{2, \$}$ \\ ${ }^{1}$ Department of Physics and Astronomy, University of California, Los Angeles, \\ Los Angeles, California 90095-1547, USA \\ ${ }^{2}$ Instituto de Física Corpuscular (IFIC), CSIC-Universitat de València, \\ Apartado de Correos 22085, E-46071 Valencia, Spain
}

(Received 3 January 2019; published 24 May 2019)

\begin{abstract}
Geoneutrinos can provide a unique insight into Earth's interior, its central engine, and its formation history. We study the detection of geoneutrinos in large direct detection experiments, which has been considered nonfeasible. We compute the geoneutrino-induced electron and nuclear recoil spectra in different materials, under several optimistic assumptions. We identify germanium as the most promising target element due to the low nuclear recoil energy threshold that could be achieved. The minimum exposure required for detection would be $\mathcal{O}(10)$ ton-years. The realistic low thresholds achievable in germanium and silicon permit the detection of ${ }^{40} \mathrm{~K}$ geoneutrinos. These are particularly important to determining Earth's formation history, but they are below the kinematic threshold of inverse beta decay, the detection process used in scintillator-based experiments.
\end{abstract}

DOI: 10.1103/PhysRevD.99.093009

\section{INTRODUCTION}

The nature of dark matter (DM) remains one of the most pressing issues in modern physics. While weakly interacting massive particles (WIMPs) remain a theoretically wellmotivated DM candidate, despite significant efforts no convincing detection signatures have been observed and a sizable portion of the allowed parameter space has been constrained. Planned next-generation large-scale direct detection experiments will further explore the uncharted corners of WIMP interaction type, candidate mass, and cross section. The experiments will eventually encounter an irreducible background due to coherent neutrino-nuclear interactions of solar as well as other neutrinos-i.e., the "neutrino floor" (e.g., Refs. [1-3]). The neutrino floor can obfuscate the potential DM signal. Hence, this could constitute a major obstacle in making definitive statements regarding DM observations. However, the neutrino signal, which experiments will surely observe, as well as possible other sources, constitute in themselves interesting targets of exploration. It is thus imperative and timely to fully explore the scientific scope of large direct detection experiments beyond their main scope as DM detectors.

\footnotetext{
*gelmini@physics.ucla.edu

vtakhist@physics.ucla.edu

sam.witte@ific.uv.es
}

Published by the American Physical Society under the terms of the Creative Commons Attribution 4.0 International license. Further distribution of this work must maintain attribution to the author(s) and the published article's title, journal citation, and DOI. Funded by SCOAP ${ }^{3}$.
The next generation of large-scale direct detection experiments constitute a promising venue as neutrino detectors. This has been bolstered by the recent detection of the coherent neutrino-nucleus scattering by the COHERENT experiment [4]. Both coherent neutrinonucleus as well as elastic electron-neutrino scatterings in direct detection experiments have already been employed in a range of studies to assess future detection capabilities within the context of neutrino-related physics, including sterile neutrinos (e.g., Refs. [5,6]), nonstandard neutrino interactions (e.g., Refs. [7,8]), supernova explosions [9], and solar neutrinos (e.g., Refs. [6,10-12]), as well as DM annihilations [13].

Geoneutrinos (see, e.g., Ref. [14] for review) are antineutrinos $\bar{\nu}_{e}$ that originate from radioactive decays of uranium ${ }^{238} \mathrm{U}$, thorium ${ }^{232} \mathrm{Th}$, and potassium ${ }^{40} \mathrm{~K}$ within the Earth's interior. They provide a unique insight into the Earth's composition and heat production mechanisms, as well as its thermal evolution and formation. In particular, geoneutrinos can reveal crucial information about the Earth's energy budget, a fundamental quantity in geology. They allow one to establish which fraction of energy originates from ongoing radiogenic decays of heatproducing elements and which is primordial, associated with Earth's formation history. Furthermore, geoneutrinos can test the hypothetical Earth's central reactor, proposed to source the Earth's magnetic field [15].

The study of geoneutrinos finally became a possibility with recent developments of neutrino detectors. Geoneutrino observations have been reported by the KamLAND [16] and Borexino experiments [17], through 
inverse beta decay (IBD) $\bar{\nu}_{e}+p \rightarrow e^{+}+n$ interactions within scintillator materials. Large uncertainties, originating from low statistics and high backgrounds, do not allow experiments to make definitive statements regarding geophysics at the current stage. Further progress is expected with future scintillator-based experiments such as SNO+ [18], JUNO [19,20], HANOHANO [21], and Jinping [22-24]. Directional detection experiments that utilize electron-neutrino scattering have also been put forward $[25,26]$.

Following earlier work in the field (e.g., Ref. [2]), a potential geoneutrino signal in direct detection experiments has been largely neglected, assumed to be too small for detection. Given the status of experimental searches and the plans for larger detectors, as well as recent interest in geoneutrinos, it is timely to revisit this issue and reassess the geoneutrino observation potential of future large-scale direct detection experiments. This is the main purpose of this work.

\section{LARGE DIRECT DETECTION EXPERIMENTS}

A variety of proposals for the next-generation multitonscale direct detection experiments have been put forth [27]. Due to the inherent uncertainty in the final designs, making definitive statements about their scientific capabilities requires making assumptions about their composition, energy threshold, and backgrounds. We explore several of the most likely detector configurations, based on xenon $(\mathrm{Xe})$, germanium $(\mathrm{Ge})$, argon (Ar), and silicon (Si), assuming optimistic design realizations.

In Table I, we list the experimental setups we consider, each with a different target element. We do not include isotopic abundances of elements that contribute less than $1 \%$. We optimistically assume that experiments have perfect detection efficiency and energy resolution. Furthermore, when considering neutrino coherent interactions with the nuclei, the expected background is assumed to arise exclusively from neutrinos. This allows us to treat all the analyses on the same footing and provide results independent of specific configurations that could change in the future. The same assumption is not realistic for neutrino-electron scattering, since the backgrounds there are expected to be significant. Thus, we do not analyze this channel.

The reactor neutrino background, as well as the geoneutrino signal, depends sensitively on the location of the experiment. We assume that the experiment is located in the Jinping Underground Laboratory in China, where the expected geoneutrino signal is the largest and the reactor background is small. Its depth (6720 m.w.e.) ensures that the background due to cosmogenic muons will be highly suppressed.

Recent analyses of xenon-based detectors, such as XENON1T [28,29] and LUX [30], set nuclear recoil thresholds of a few $\mathrm{keV}$, when employing both scintillation
TABLE I. Considered experimental configurations. The last column shows the maximum energy of our region of interest.

\begin{tabular}{|c|c|c|c|c|}
\hline Target material & $A(Z)$ & $\begin{array}{l}\text { Isotope } \\
\text { fraction }\end{array}$ & $\begin{array}{c}\text { Energy } \\
\text { threshold } \\
{[\mathrm{eVnr}]}\end{array}$ & $\begin{array}{c}\text { Max } \\
\text { energy } \\
\text { [eVnr] }\end{array}$ \\
\hline xenon $(\mathrm{Xe})$ & $\begin{array}{l}128(54) \\
129(54) \\
130(54) \\
131(54) \\
132(54) \\
134(54) \\
136(54)\end{array}$ & $\begin{array}{l}0.019 \\
0.264 \\
0.041 \\
0.212 \\
0.269 \\
0.104 \\
0.089\end{array}$ & 100 & 267 \\
\hline argon (Ar) & $40(18)$ & 0.996 & 100 & 855 \\
\hline germanium $(\mathrm{Ge})$ & $\begin{array}{l}70(32) \\
72(32) \\
73(32) \\
74(32) \\
76(32)\end{array}$ & $\begin{array}{l}0.208 \\
0.275 \\
0.077 \\
0.363 \\
0.076\end{array}$ & 40 & 489 \\
\hline silicon $(\mathrm{Si})$ & $\begin{array}{l}28(14) \\
29(14) \\
30(14)\end{array}$ & $\begin{array}{l}0.922 \\
0.047 \\
0.031\end{array}$ & 78 & 1221 \\
\hline
\end{tabular}

and ionization signals. These experiments can go to even lower recoil energies when using only their ionization signal, although their background rejection in this operation mode is less efficient. In this way the XENON100 Collaboration lowered its threshold to $0.7 \mathrm{keV}$ for nuclear recoils [31]. Thresholds as low as $0.1 \mathrm{keV}$ have been used in some projections for future detectors, such as LZ [32]. Similarly, the argon-based DarkSide-50 was able to lower its threshold for nuclear recoils to $0.6 \mathrm{keV}$ with an analysis of only the ionization signal [33] (although their official analysis has somewhat higher thresholds [34]). Assuming optimistic realizations of future noble-gas-based experiments, we use a threshold of $0.1 \mathrm{keV}$ for xenon as well as argon.

Germanium and silicon will both be used in the future SuperCDMS SNOLAB experiment. With their HV (highvoltage) detectors, SuperCDMS has the capacity to reach the nuclear recoil thresholds of $40 \mathrm{eV}$ for germanium and $78 \mathrm{eV}$ for silicon (see Table VII of Ref. [35]). We adopt these values here.

In order to maximize the geoneutrino signal when studying nuclear recoils, we will use data within a region of interest (ROI) defined by the recoil energies between the assumed experimental threshold energy and the maximum energy that can be imparted by any geoneutrino (see Table I).

\section{GEONEUTRINO SIGNAL AND BACKGROUND}

The geoneutrino signal, as well as reactor and solar neutrino background components, are displayed in Fig. 1. We describe them in more detail in the section below. 


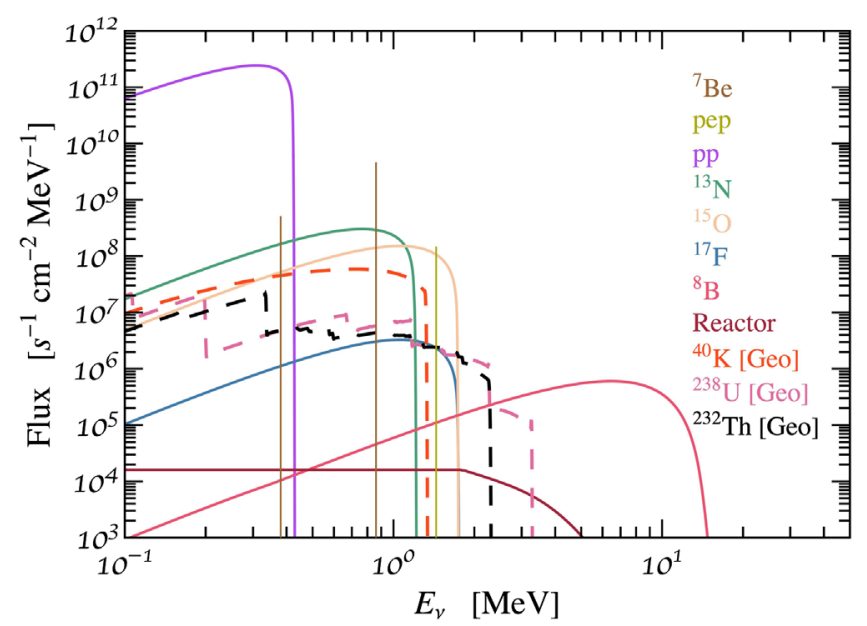

FIG. 1. Neutrino flux components for the Jinping location. The geoneutrino signal as well as solar and reactor neutrino backgrounds are shown.

\section{A. Geoneutrino signal}

\section{Spectrum}

The radioactive decays of ${ }^{238} \mathrm{U},{ }^{232} \mathrm{Th}$, and ${ }^{40} \mathrm{~K}$ are responsible for more than $99 \%$ of the Earth's radiogenic heat. These elements decay via a series of $\alpha$ and $\beta^{-}$ processes, which terminate at stable isotopes. Each $\beta^{-}$ decay results in the emission of $\bar{\nu}_{e}$. Comprehensive computations are needed to obtain the overall resulting $\bar{\nu}_{e}$ spectrum for each decay chain, including the spectral shapes and rates of individual decays for more than 80 different branches in each chain [36]. In Fig. 2 we display the resulting geoneutrino spectra for each element used in our study, as shown in Ref. [16]. Note that the kinematic threshold of $1.8 \mathrm{MeV}$ for inverse beta decay (IBD)

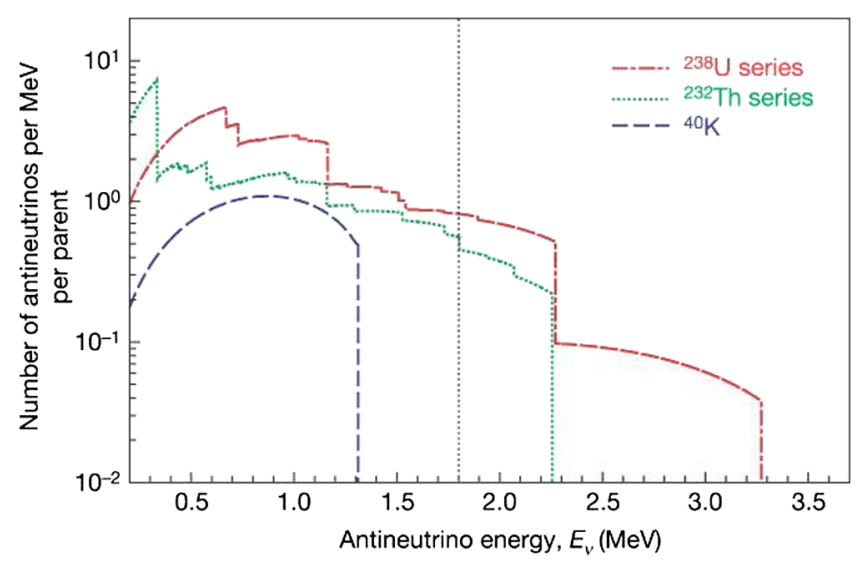

FIG. 2. The geoneutrino $\bar{\nu}_{e}$ spectrum expected from ${ }^{238} \mathrm{U},{ }^{232} \mathrm{Th}$, and ${ }^{40} \mathrm{~K}$ decay chains. The dotted vertical line at $1.8 \mathrm{MeV}$ represents the kinematic threshold for IBD interactions. Reproduced from Ref. [16]. interactions makes detectors based on this reaction insensitive to geoneutrinos generated from the ${ }^{40} \mathrm{~K}$ decays.

\section{Flux normalization}

The geoneutrino flux expected in different locations strongly depends on the amount and distribution of heatproducing elements within Earth. The Earth's surface heat flow of $47 \pm 2 \mathrm{TW}$ [37] is composed partly of heat generated by radioactive decays in the crust and mantle and partly of heat from the secular planet cooling (i.e., primordial heat that is a byproduct of the Earth's formation). While the crust contributions are relatively well known, the contributions from the mantle remain highly uncertain. The predicted radiogenic heat, originating from the heat-producing elements, varies greatly between different bulk silicate Earth models [38], which do not include the Earth's metallic core. The models can be generally classified as cosmochemical, geochemical, and geodynamical, which make radiogenic heat predictions of $11 \pm 2$, $17 \pm 3$, and $33 \pm 3 \mathrm{TW}$, respectively.

To maximize the geoneutrino signal, we consider the most optimistic scenario of $100 \%$ of Earth's heat being radiogenic-i.e., Earth's radiogenic heat comprising $\sim 47$ TW [14]. This choice is compatible with current geoneutrino measurements within a few standard deviations [39]. The location-dependent geoneutrino flux is particularly sensitive to the amount of crust beneath the laboratory site, which contains the largest portion of heatproducing elements. Due to this reason, as well as the substantial distance to the closest nuclear reactors, Jinping Underground Laboratory, situated near the Himalayan mountains in China where the amount of crust is maximal, has been previously identified as a favorable location for geoneutrino detection [24]. In principle, the geoneutrino flux is calculated by summing contributions from each Earth grid component, using a detailed geological map specifying heat-producing element distribution and abundance. Assuming a nearly fully radiogenic Earth, we take the geoneutrino flux at Jinping (see Fig. 6 of Ref. [23]) corresponding to $\gtrsim 90 \mathrm{TNU}$ (terrestrial natural units), which is 2.5 larger (see Fig. 2 of Ref. [24]) than the computed flux for each radiogenic component at the SNOLab (Sudbury, Canada) using the reference Earth model (see Table 2 of Ref. [40]). The geoneutrino fluxes we adopt are provided in Table II. The corresponding flux uncertainties are taken from Ref. [40].

TABLE II. Geoneutrino signal flux.

\begin{tabular}{lcc}
\hline \hline $\begin{array}{l}\text { Neutrino } \\
\text { component }\end{array}$ & Flux $\left[\mathrm{cm}^{2} \mathrm{~s}^{-1}\right]$ & Max energy $\mathrm{E}_{\nu}[\mathrm{MeV}]$ \\
\hline Geo $\left(\bar{\nu}_{e},{ }^{40} \mathrm{~K}\right)$ & $5.47(1 \pm 0.006) \times 10^{7}$ & 1.32 \\
Geo $\left(\bar{\nu}_{e},{ }^{238} \mathrm{U}\right)$ & $1.14(1 \pm 0.300) \times 10^{7}$ & 3.99 \\
Geo $\left(\bar{\nu}_{e},{ }^{232} \mathrm{Th}\right)$ & $1.14(1 \pm 0.300) \times 10^{7}$ & 2.26 \\
\hline \hline
\end{tabular}




\section{B. Neutrino background}

Since the geoneutrino signal ceases at neutrino energies above $\sim 5 \mathrm{MeV}$, we shall only consider the backgrounds due to solar and reactor neutrinos (see Fig. 1), which dominate at energies $E_{\nu} \lesssim 20 \mathrm{MeV}$. At higher neutrino energies, contributions from atmospheric and diffuse supernova neutrinos also become important.

\section{Solar neutrinos}

Solar electron neutrinos $\nu_{e}$ are produced as a byproduct of nuclear fusion reactions in the Sun (see Ref. [41] for review). Multiple reaction chains contribute, varying in resulting neutrino flux and energy. More than $98 \%$ of the Sun's energy is produced through the proton-proton cycle. Starting with the initial reaction of $p+p \rightarrow{ }^{2} \mathrm{H}+e^{+}+\nu_{e}$, this cycle results in $p p$, hep, pep, ${ }^{7} \mathrm{Be}$, and ${ }^{8} \mathrm{~B}$ neutrino components. The remaining solar energy is released through the carbon-nitrogen-oxygen (CNO) cycle, which results in ${ }^{13} \mathrm{~N},{ }^{15} \mathrm{O}$, and ${ }^{17} \mathrm{~F}$ neutrinos. The solar neutrino background is independent of the laboratory location.

Specific solar neutrino fluxes can be inferred from a solar model. The standard solar model (GS98) [42] showed a good agreement with helioseismological studies. On the other hand, more recent constructions (AGSS09) [43] that possess a higher degree of internal consistency appear to disagree with results from helioseismology. This discrepancy is the so-called "solar metallicity" problem. The CNO neutrinos are particularly promising in this context, given their ability to shed light on the Sun's internal composition, and their observation potential in future direct detection experiments has been recently explored $[6,10,12]$. For our analysis we assume the solar flux normalizations and uncertainties as predicted by AGSS09 (see Table 2 of Ref. [41]), which we specify in Table III. The background in our study is dominated by ${ }^{8} \mathrm{~B}$ neutrinos. Since the ${ }^{8} \mathrm{~B}$ flux difference between the two solar models is only $\sim 20 \%$, we do not expect a significant change in our sensitivity results if the alternative model is employed.

TABLE III. Solar and reactor neutrino background fluxes.

\begin{tabular}{llc}
\hline \hline Neutrino component & \multicolumn{1}{c}{ Flux $\left[\mathrm{cm}^{-2} \mathrm{~s}^{-1}\right]$} & $\begin{array}{c}\text { Max energy } \\
\mathrm{E}_{\nu}[\mathrm{MeV}]\end{array}$ \\
\hline Solar $\left(\nu_{e}, \mathrm{pp}\right)$ & $6.03(1 \pm 0.006) \times 10^{10}$ & 0.42 \\
Solar $\left(\nu_{\mathrm{e}}, \mathrm{pep}[\right.$ line $\left.]\right)$ & $1.47(1 \pm 0.012) \times 10^{8}$ & 1.45 \\
Solar $\left(\nu_{e}\right.$, hep $)$ & $8.31(1 \pm 0.300) \times 10^{3}$ & 18.77 \\
Solar $\left(\nu_{e},{ }^{7} \mathrm{Be}[\right.$ line 1$\left.]\right)$ & $4.56(1 \pm 0.070) \times 10^{8}$ & 0.39 \\
Solar $\left(\nu_{e},{ }^{7} \mathrm{Be}[\right.$ line 2] $\left.]\right)$ & $4.10(1 \pm 0.070) \times 10^{9}$ & 0.87 \\
Solar $\left(\nu_{e},{ }^{8} \mathrm{~B}\right)$ & $4.59(1 \pm 0.140) \times 10^{6}$ & 16.80 \\
Solar $\left(\nu_{e},{ }^{13} \mathrm{~N}\right)$ & $2.17(1 \pm 0.140) \times 10^{8}$ & 1.20 \\
Solar $\left(\nu_{e},{ }^{15} \mathrm{O}\right)$ & $1.56(1 \pm 0.150) \times 10^{8}$ & 1.73 \\
Solar $\left(\nu_{e},{ }^{17} \mathrm{~F}\right)$ & $3.40(1 \pm 0.170) \times 10^{6}$ & 1.74 \\
Reactor $\left(\bar{\nu}_{\mathrm{e}}\right)$ & $4.95(1 \pm 0.100) \times 10^{4}$ & 10.00 \\
\hline \hline
\end{tabular}

\section{Reactor neutrinos}

Reactor electron antineutrinos $\bar{\nu}_{e}$ (see Ref. [44] for a review) originate from fission $\beta$ decays of uranium ${ }^{235} \mathrm{U}$ and $\left.{ }^{238} \mathrm{U}\right)$ and plutonium $\left({ }^{239} \mathrm{Pu}\right.$ and $\left.{ }^{241} \mathrm{Pu}\right)$ within the reactor fuel. Since the considered isotopes are short lived, the corresponding neutrino flux follows the reactor operation. The fraction of isotope contributions as well as the average energy release per fission are given in Table IV, following Ref. [45]. As a reactor operates, its fuel composition and relative isotope fractions change with time, in a manner specific for each individual reactor. We neglect these effects (an analysis including these contributions can be found in Ref. [46]).

Unlike the flux of solar neutrinos, the reactor neutrino flux depends on the laboratory location, and in particular on the characteristics and distances of the main contributing reactors [47]. The flux of reactor antielectron neutrinos from an isotope $k$ is given by

$$
\phi_{k}(E)=\frac{R_{\bar{\nu}_{e}}}{4 \pi d^{2}} \sum_{k} f_{k} S_{k}(E)
$$

where $R_{\bar{\nu}_{e}}$ is the emission rate, $d$ is the distance of a given reactor to the laboratory, $S_{k}(E)$ is the corresponding neutrino spectrum, and $f_{k}$ is the fraction of the isotope $k$ in the reactor fuel. The neutrino emission rate is

$$
R_{\bar{\nu}_{e}}=N_{\nu, \mathrm{f}} \frac{P_{\mathrm{th}}}{\sum_{k} f_{k} E_{k}} e \simeq 6 \times 10^{20}\left(\frac{P_{\mathrm{th}}}{1 \mathrm{GW}}\right) e \mathrm{~s}^{-1},
$$

where $N_{\nu, \mathrm{f}} \simeq 6$ is the average number of antineutrinos produced per fission, $P_{\text {th }}$ is the thermal power output of the reactor, $E_{k}$ is the fission energy, and $e=0.75(1 \pm 0.1)$ is the average reactor operation efficiency that includes shutdowns [48]. The uncertainty of the efficiency reflects an uncertainty of about $8 \%$ in the reactor antineutrino spectrum (see Table 3 of Ref. [49]) as well as an uncertainty of a few percent on the fission emission. This is the source of the reactor flux uncertainty in Table III. Approximate analytic expressions for neutrino spectra $S_{k}\left(E_{\nu}\right)$ have been constructed [46,50-52]. We adopt the spectra from the model of Ref. [51], which is based on a phenomenological fit to data with an exponentiated polynomial. The resulting expression is

TABLE IV. Fission fraction and average released energy for each nuclear reactor isotope.

\begin{tabular}{lcc}
\hline \hline Isotope & $f_{k}$ & $E_{k}[\mathrm{MeV} /$ fission $]$ \\
\hline${ }^{235} \mathrm{U}$ & 0.58 & $202.36 \pm 0.26$ \\
${ }^{238} \mathrm{U}$ & 0.07 & $205.99 \pm 0.52$ \\
${ }^{239} \mathrm{Pu}$ & 0.30 & $211.12 \pm 0.34$ \\
${ }^{241} \mathrm{Pu}$ & 0.05 & $214.26 \pm 0.33$ \\
\hline \hline
\end{tabular}


TABLE V. Fitted values of the reactor neutrino spectrum $\alpha_{i, k}$ coefficients, for $i=1$ to 6 , for the dominant nuclear isotopes (denoted by $k$ ).

\begin{tabular}{lcccc}
\hline \hline$i$ & ${ }^{235} \mathrm{U}$ & ${ }^{238} \mathrm{U}$ & ${ }^{239} \mathrm{Pu}$ & ${ }^{241} \mathrm{Pu}$ \\
\hline 1 & 3.217 & 0.4833 & 6.413 & 3.251 \\
2 & -3.111 & 0.1927 & -7.432 & -3.204 \\
3 & 1.395 & -0.1283 & 3.535 & 1.428 \\
4 & -0.3690 & -0.006762 & -0.8820 & -0.3675 \\
5 & 0.04445 & 0.002233 & 0.1025 & 0.04254 \\
6 & -0.00253 & -0.0001536 & -0.004550 & -0.001896 \\
\hline \hline
\end{tabular}

$$
S_{k}\left(E_{\nu}\right)=\frac{d N_{\nu}}{d E_{\nu}}=\exp \left(\sum_{i=1}^{6} \alpha_{i, k} E_{\nu}^{i-1}\right)
$$

where $\alpha_{i, k}$ is the respective fit coefficient of order $i$. The best values of the fit coefficients, as obtained in Ref. [51], are displayed in Table V. Strictly, these fits are only valid for $E_{\nu} \gtrsim 1.8 \mathrm{MeV}$. For uranium, Ref. [50] does not find substantial deviations in the fit results for energies above $0.5 \mathrm{MeV}$. For other elements, the flux at lower energies appears enhanced. Since this could be a model artifact and not of physical significance, we conservatively set $S_{k}\left(E_{\nu}<1.8 \mathrm{MeV}\right)=S_{k}\left(E_{\nu}=1.8 \mathrm{MeV}\right)$ for all elements. This effectively appears as a "kink" feature visible in the resulting flux figures. Recent measurements of the reactor antineutrino spectra from Daya Bay [53] indicate agreement with the predictions of the leading theoretical models of Huber-Muller [51,52] and ILL-Vogel [54-57] at the level of $\sim 10 \%$ difference on the predicted antineutrino yield. Since reactor background constitutes a subdominant contribution in our study, we do not expect reactor neutrino modeling uncertainty to significantly affect our results.

We list the reactors considered in our study for the Jinping location in Table VI.

\section{Recoil rates}

We consider two types of interactions in direct detection experiments: elastic electron neutrino scattering and coherent neutrino-nucleus scattering.

For a neutrino flux $\phi_{\nu}\left(E_{\nu}\right)$, the resulting differential event rate per unit time and detector mass as a function of the recoil energy $E_{R}$ per unit time and mass $m_{I}$ of a target nuclide $I$ in a detector is given by

$$
\frac{d R_{\nu}^{I}}{d E_{R}}=\frac{C_{I}}{m_{I}} \int_{E_{\nu}^{\min }} \phi_{\nu}\left(E_{\nu}\right) \frac{d \sigma^{I}\left(E_{\nu}, E_{R}\right)}{d E_{R}} d E_{\nu}
$$

where $d \sigma^{I}\left(E_{\nu}, E_{R}\right) / d E_{R}$ is the coherent neutrino-nucleus scattering differential cross section and $C_{I}$ is the fraction of nuclide $I$ in the material. When several nuclides are present, for each type of neutrino flux contribution $\phi_{\nu}\left(E_{\nu}\right)$ the differential event rate is given by summing over them:
TABLE VI. List of selected most relevant nuclear reactors for Jinping. Columns contain, from left to right, the name of the reactor, the GPS location, the distance to the laboratory in kilometers, and the thermal output of the reactor in MW.

\begin{tabular}{lccr}
\hline \hline Reactor & Location & $\begin{array}{c}\text { Distance } \\
{[\mathrm{km}]}\end{array}$ & $\begin{array}{r}\text { Output } \\
{\left[\mathrm{MW}_{t}\right]}\end{array}$ \\
\hline Fangchengang & $\begin{array}{c}21^{\circ} 40^{\prime} 15^{\prime \prime} \mathrm{N} \\
108^{\circ} 33^{\prime} 30^{\prime \prime} \mathrm{E}\end{array}$ & 998 & 12110 \\
& $\begin{array}{l}19^{\circ} 27^{\prime} 39^{\prime \prime} \mathrm{N} \\
108^{\circ} 53^{\prime} 60^{\prime \prime} \mathrm{E}\end{array}$ & 1206 & 3806 \\
Changjiang & $21^{\circ} 42^{\prime} 30^{\prime \prime} \mathrm{N}$ & 1239 & 17430 \\
& $112^{\circ} 15^{\prime} 40^{\prime \prime} \mathrm{E}$ & & \\
Yangjiang & $21^{\circ} 55^{\prime} 04^{\prime \prime} \mathrm{N}$ & 1318 & 1980 \\
& $112^{\circ} 58^{\prime} 55^{\prime \prime} \mathrm{E}$ & & \\
Taishan & $22^{\circ} 36^{\prime} 17^{\prime \prime} \mathrm{N}$ & 1422 & 11620 \\
& $114^{\circ} 33^{\prime} 05^{\prime \prime} \mathrm{E}$ & & \\
Ling Ao & $25^{\circ} 26^{\prime} 45^{\prime \prime} \mathrm{N}$ & 1763 & 17740 \\
& $119^{\circ} 26^{\prime} 50^{\prime \prime} \mathrm{E}$ & & \\
Fuqing & $35^{\circ} 24^{\prime} 54^{\prime \prime} \mathrm{N}$ & 2463 & 16874 \\
& $126^{\circ} 25^{\prime} 26^{\prime \prime} \mathrm{E}$ & & \\
Hanbit & & & \\
& &
\end{tabular}

$$
\frac{d R_{\nu}}{d E_{R}}=\mathrm{MT} \sum_{I} \frac{d R_{\nu}^{I}}{d E_{R}}
$$

where MT is the experimental exposure.

A similar expression holds for electrons if we neglect the effects of their atomic and molecular binding. In the approximation of considering electrons as free in the material, we need to take into account the charge number $Z_{I}$ in every nuclide $I$. Hence, the interaction rate in a detector becomes

$\frac{d R_{\nu}}{d E_{R}}=\mathrm{MT} \sum_{I} \frac{C_{I}}{m_{I}} Z_{I} \int_{E_{\nu}^{\min }} \phi_{\nu}\left(E_{\nu}\right) \frac{d \sigma^{e}\left(E_{\nu}, E_{R}\right)}{d E_{R}} d E_{\nu}$,

where $d \sigma^{e}\left(E_{\nu}, E_{R}\right) / d E_{R}$ is the neutrino-electron elastic scattering differential cross section.

For a target mass $m$ at rest $\left(m_{I}\right.$ or the electron mass $\left.m_{e}\right)$, the minimum neutrino energy required to produce a recoil of energy $E_{R}$ is

$$
E_{\nu}^{\min }=\sqrt{\frac{m E_{R}}{2}} .
$$

The maximum recoil energy due to a collision with a neutrino of energy $E_{\nu}$ is

$$
E_{R}^{\max }=\frac{2 E_{\nu}^{2}}{m+2 E_{\nu}} .
$$

\section{Coherent nuclear scattering cross section}

The Standard Model coherent-scattering neutrinonucleus cross section is given by [58] 


$$
\frac{d \sigma^{I}\left(E_{\nu}, E_{R}\right)}{d E_{R}}=\frac{G_{f}^{2} m_{I}}{4 \pi} Q_{w}^{2}\left(1-\frac{m_{I} E_{R}}{2 E_{\nu}^{2}}\right) F_{\mathrm{S}, I}^{2}\left(E_{R}\right),
$$

where $m_{I}$ is the target nuclide mass; $G_{f}$ is the Fermi coupling constant; $F_{\mathrm{SI}, I}\left(E_{R}\right)$ is the form factor, which we take to be the Helm form factor [59]; $Q_{w}=[(1-$ $\left.\left.4 \sin ^{2} \theta_{\mathrm{W}}\right) Z_{I}-N_{I}\right]$ is the weak nuclear charge; $N_{I}$ is the number of neutrons, $Z_{I}$ is the number of protons; and $\theta_{\mathrm{W}}$ is the Weinberg angle. Since $\sin ^{2} \theta_{\mathrm{W}}=0.223$ [60], the coherent neutrino-nucleus scattering cross section follows an approximate $N_{I}^{2}$ scaling.

\section{Elastic electron scattering cross section}

The Standard Model neutrino-electron scattering crosssection is [50]

$\frac{d \sigma^{e}\left(E_{\nu}, E_{R}\right)}{d E_{R}}=\frac{G_{f}^{2} m_{e}}{2 \pi}\left[g_{\nu}^{2}+g_{\nu}^{\prime 2}\left(1-\frac{E_{R}}{E_{\nu}}\right)^{2}+g_{\nu} g_{\nu}^{\prime} \frac{m_{e} E_{R}}{E_{\nu}^{2}}\right]$.

Here, the flavor-dependent $g_{\nu}$ weak couplings

$$
g_{\nu(\mu, \tau)}=2 \sin ^{2} \theta_{\mathrm{W}}-1, \quad g_{\nu(e)}=2 \sin ^{2} \theta_{\mathrm{W}}+1
$$

are enhanced for the electron flavor, while

$$
g_{\nu}^{\prime}(e, \mu, \tau)=2 \sin ^{2} \theta_{\mathrm{W}} .
$$

The corresponding antineutrino cross-sections are obtained via $g_{\bar{\nu}(\nu)} \leftrightarrow g_{\nu(\bar{\nu})}^{\prime}$ interchange.

The electron-neutrino cross section of Eq. (10) is valid only for a neutrino scattering with a free electron. However, the target electrons of interest in this study are bound to nuclei. For larger recoil energies and smaller nuclei, the atomic effects are expected to become negligible. On the

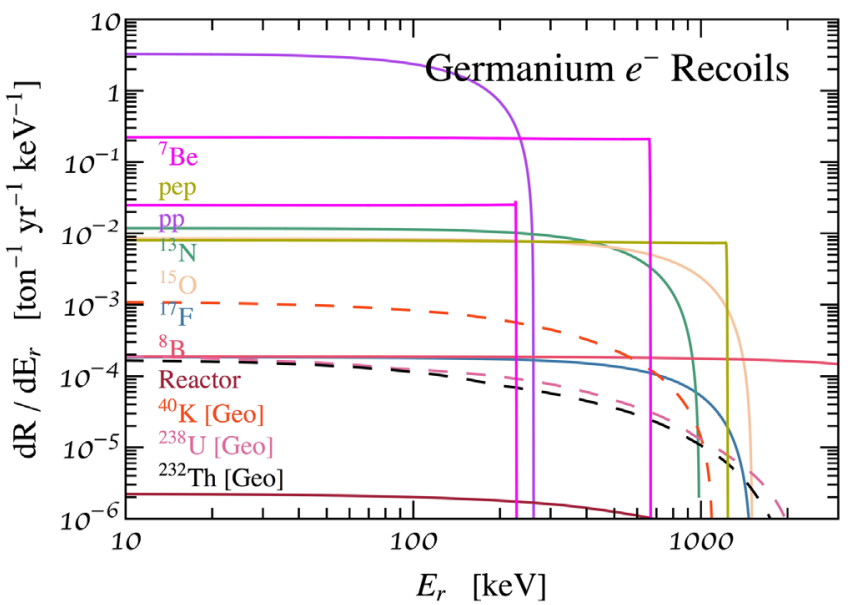

other hand, for recoil energies of a few $\mathrm{keV}$ in germanium [61-63] and $\mathcal{O}(10) \mathrm{keV}$ in xenon [64], the cross sections can become noticeably suppressed. Since we do not perform a statistical analysis for electron recoils, this effect is neglected.

\section{Neutrino oscillations}

The coherent neutrino-nucleus scattering is mediated by the $Z$ boson. Thus, this interaction is independent of the neutrino flavor and the corresponding oscillation effects. On the other hand, electron-neutrino interactions are sensitive to oscillation effects.

Due to the long range of production distances, the survival probability of $\bar{\nu}_{e}$ geoneutrinos due to averaged out oscillation effects is approximately

$\left\langle P_{e e}\right\rangle=\cos ^{4} \theta_{13}\left(1-\frac{\sin ^{2}\left(2 \theta_{12}\right)}{2}\right)+\sin ^{4} \theta_{13} \simeq 0.55$

assuming $\theta_{13}=8.5^{\circ}$ and $\theta_{12}=34.5^{\circ}$ [65]. The Earth's Mikheyev-Smirnov-Wolfenstein (MSW) oscillation effects for geoneutrinos are negligible [23]. On average, $\left\langle P_{e e}\right\rangle \simeq$ 0.55 is also valid for reactor neutrinos. For solar neutrinos we also take an electron neutrino survival probability of $\left\langle P_{e e}\right\rangle \simeq 0.55$ for all fluxes aside from ${ }^{8} \mathrm{~B}$, since they lie in the region of vacuum oscillations of $E_{\nu} \lesssim 1 \mathrm{MeV}$. For ${ }^{8} \mathrm{~B}$ neutrinos we take $\left\langle P_{e e}\right\rangle \simeq 0.35$, since their energies are in the matter-enhanced oscillation region of $E_{\nu} \gtrsim 5 \mathrm{MeV}$. This choice is consistent with the large mixing angle (LMA-)MSW solution, as indicated by measurements [66].

\section{PREDICTED RECOIL SPECTRA}

\section{A. Electron recoils}

The left panel of Fig. 3 shows the recoil spectrum of the three types of geoneutrinos in germanium (dashed lines). The electron recoil spectrum is almost the same for all

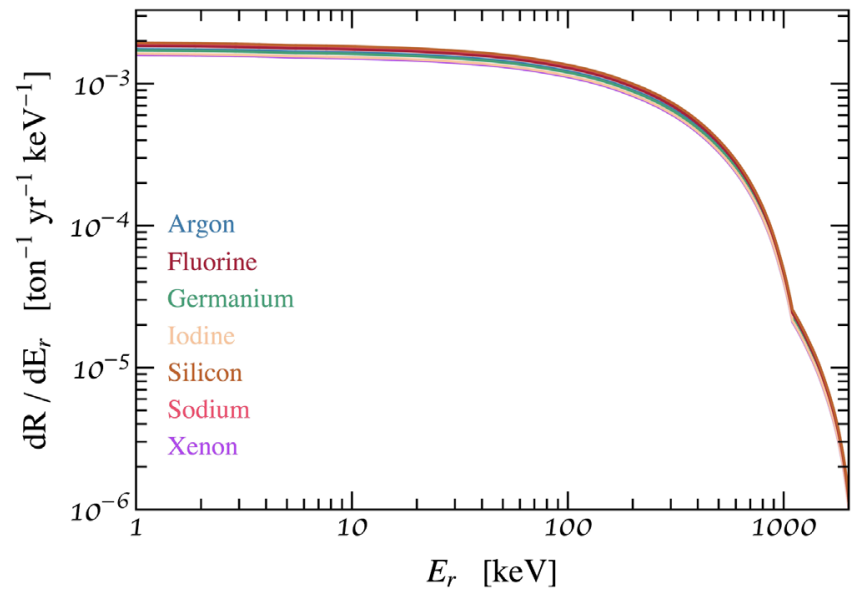

FIG. 3. Left: Electron recoil spectrum due to the indicated type of neutrinos for germanium. Right: Total geoneutrino electron recoil spectra for various target materials. 

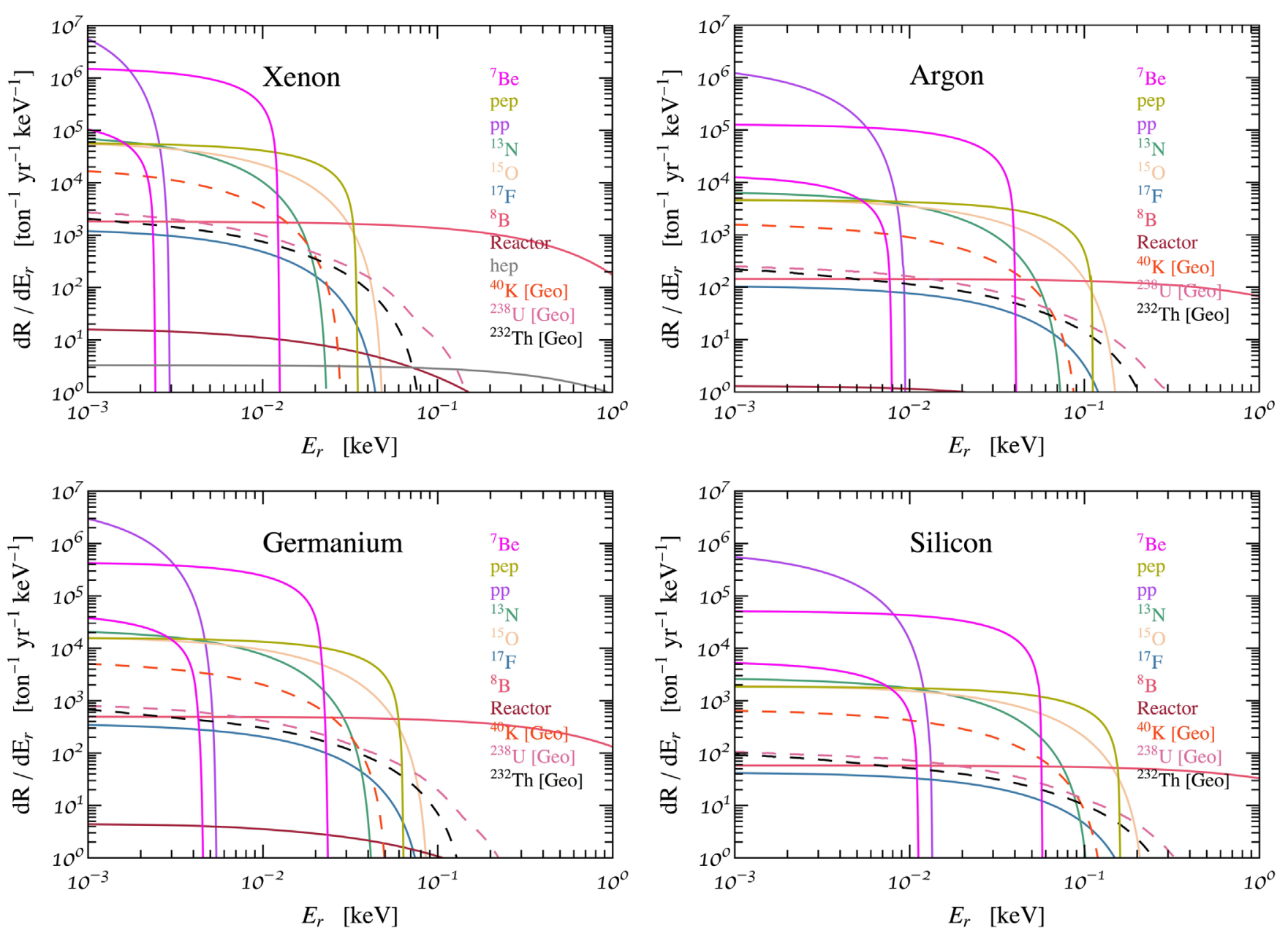

FIG. 4. Nuclear recoil spectra due to the indicated types of neutrinos for xenon, argon, germanium, and silicon.

target elements. This can be seen in the right panel of the same figure, where we show the total electron recoil spectrum (the sum of all three geoneutrino contributions) for several elements. The reason is that the ratio $Z_{I} / m_{I} \simeq Z_{I} / A_{I} m_{p}$, where $m_{p}$ is the proton mass, which enters into the rate, is approximately $1 / 2 m_{p}$ for all elements, and the differential cross section Eq. (10) is the same, neglecting binding effects.

The backgrounds for a geoneutrino signal in electron recoils due to contributions other than neutrinos are expected to be large. Thus, we do not examine the detectability of geoneutrinos through electron scattering.

\section{B. Nuclear recoils}

Figure 4 shows the three geoneutrino nuclear recoil spectra (dashed lines) for $\mathrm{Xe}, \mathrm{Ar}, \mathrm{Ge}$, and $\mathrm{Si}$. The total (sum of all three contributions) geoneutrino nuclear recoil spectra for these and other target elements are shown in Fig. 5. These spectra, and the corresponding differential cross sections, differ considerably between various elements. With the threshold we are using (see Table I), Fig. 4 shows that a ${ }^{40} \mathrm{~K}$ geoneutrino signal cannot be detected in either Xe or Ar, and that a ${ }^{232} \mathrm{Th}$ signal cannot be observed in Xe. The lowest threshold of $0.6 \mathrm{keV}$ currently employed in Xe-based experiments would not allow us to detect any geoneutrino signal. The most promising targets for

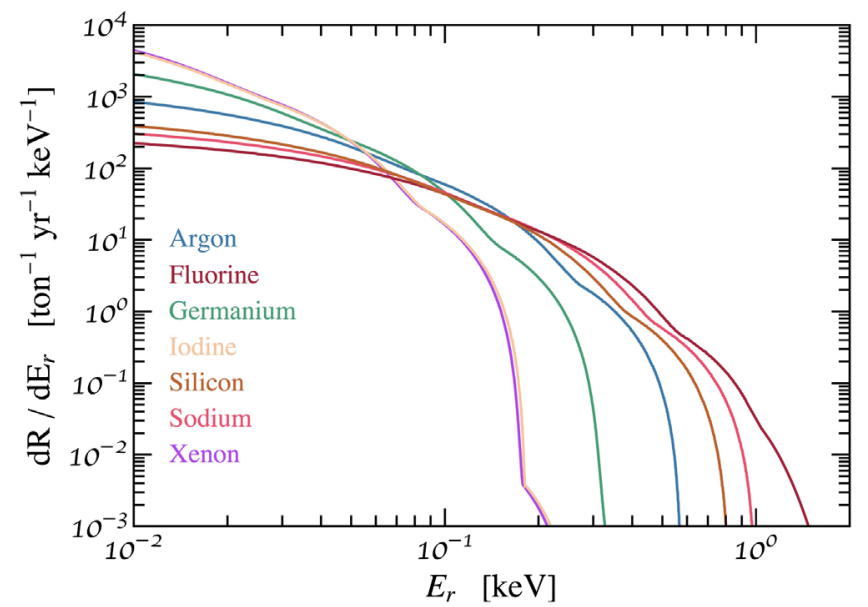

FIG. 5. Total geoneutrino nuclear recoil spectra for various target materials. 
detecting geoneutrinos are therefore $\mathrm{Ge}$ and $\mathrm{Si}$, because of the achievable low threshold as demonstrated by the SuperCDMS SNOLAB HV (high-voltage) detectors.

\section{DETECTION SENSITIVITY FOR NUCLEAR RECOILS}

\section{A. Statistical analysis}

To determine the discovery sensitivity of a direct detection experiment, we use a frequentist analysis based on the profile likelihood ratio test [67-69] used recently in Ref. [3] and other studies [1,70-72]. This test is performed by generating simulated datasets for the experiment, assuming a normalization (i.e., an energy integrated neutrino flux) for each of the considered neutrino fluxes $\phi_{\nu_{k}}$, which we take here to be the average theoretical predicted value $\bar{\phi}_{\nu_{k}}$. Here $k=1,2, \ldots, n_{\nu}$, with $n_{\nu}$ being the total number of neutrino contributions in Table III as well as the geoneutrino fluxes in Table II that produce recoils above the particular experimental threshold. More specifically, for each of the $\phi_{\nu_{k}}$ parameters we generate fake data consisting of a total number of "observed" events $N_{\mathrm{o}}$ at particular recoil energies $E_{j}$ with $j=1,2, \ldots, N_{\mathrm{o}}$ that we use to define the following likelihood function:

$$
\begin{aligned}
\mathcal{L}= & \left\{\frac{e^{-N_{\mathrm{E}}}}{N_{\mathrm{o}} !} \prod_{j=1}^{N_{\mathrm{o}}} \operatorname{MT}\left(\left.\frac{d R_{\mathrm{tot}}}{d E_{R}}\right|_{E_{\mathrm{R}}=E_{j}}\right)\right\} \\
& \times \prod_{k^{\prime}=1}^{n_{\nu}-n_{g}} \frac{1}{\sqrt{2 \pi} \sigma_{\nu_{k^{\prime}}}} \exp \left[-\left(\frac{\phi_{\nu_{k^{\prime}}}-\bar{\phi}_{\nu_{k^{\prime}}}}{\sqrt{2} \sigma_{\nu_{k^{\prime}}}}\right)^{2}\right] .
\end{aligned}
$$

Here, $n_{g}$ is the number of geoneutrino species that produce recoils above threshold and $k^{\prime}$ numbers the fluxes of neutrinos other than geoneutrinos, with uncertainties $\sigma_{\nu_{k^{\prime}}}$ taken into account. The total differential rate is the sum of the contributions of the signal due to geoneutrinos, denoted by the index $k_{g}$, and the background, assumed here to be due only to all other types of neutrinos,

$$
\frac{d R_{\text {tot }}}{d E_{R}}=\sum_{k_{g}}^{n_{g}} \frac{d R_{\nu_{k_{g}}}}{d E_{\mathrm{R}}}\left(\phi_{\nu_{k g}}\right)+\sum_{k^{\prime}}^{n_{\nu}-n_{g}} \frac{d R_{\nu_{k^{\prime}}}}{d E_{\mathrm{R}}}\left(\phi_{\nu_{k^{\prime}}}\right),
$$

defined in Eq. (5). The total number of predicted events $N_{\mathrm{E}}$ is obtained by integrating Eq. (15) over our recoil energy ROI and multiplying by the assumed exposure MT. In Eq. (14), the extended likelihood (i.e., the term in the curly brackets) is multiplied by a Gaussian product of likelihoods, centered around the mean predicted flux normalization $\bar{\phi}_{\nu_{k^{\prime}}}$, for each background neutrino species $\nu_{k^{\prime}}$ $\left(k^{\prime}=1,2, \ldots, n_{\nu}-n_{g}\right)$ to take into account the uncertainty in the $\phi_{\nu_{k^{\prime}}}$ fluxes. In the Gaussian likelihoods, the $\sigma_{\nu_{k^{\prime}}}$ adopted is the $1 \sigma$ uncertainty in the particular flux given in Table III (see Sec. III B).
As explained in Ref. [3], the procedure we follow to obtain each set of simulated data involves two steps: (1) find the total number of events of each type $k$ (where $k$ numbers all neutrino types above threshold), and (2) find the recoil energy for each of the fake data events. For each realization of fake data, the number of events of a specific type, $n_{k}$, is found from a Poisson distribution $P_{k}$,

$$
P_{k}=\frac{\mu_{k}^{n_{k}} e^{-\mu_{k}}}{n_{k} !},
$$

where the mean $\mu_{k}$ is the number of events predicted by the model being tested, defined by the particular average total neutrino flux $\bar{\phi}_{\nu_{k}}$, the target element, energy range, and assumed exposure MT. Choosing a random number for the cumulative probability distribution of each $P_{k}$, one value of $n_{k}$ is randomly generated, according to an inverse transform sampling. The total number $N_{0}$ of "observed" events is then $N_{0}=\sum_{k=1}^{n_{\nu}} n_{k}$.

To determine the energy of each one of the $n_{k}$ events of each neutrino type, we use as a probability density function (PDF) the corresponding differential recoil rate $d R_{k} / d E_{R}$ normalized by the total rate (i.e., the rate integrated over the specified energy ROI for the given experiment $R_{k}$ ) as

$$
(\mathrm{PDF})_{k}=\frac{1}{R_{k}} \frac{d R_{k}}{d E_{R}}
$$

The corresponding $n_{k}$ recoil energies are again obtained with inverse transform sampling. With the above procedure, we simulate 600 datasets for each exposure and experimental configuration.

For each simulated dataset we define a test statistic $q_{0}$ that allows one to reject the background-only hypothesis $H_{0}$, assuming it is true, with a probability not larger than some value $\alpha$ that denotes the significance level of the test. In our case, $H_{0}$ is the assumption that the flux of geoneutrinos is zero, $\phi_{\nu_{k g}}=0$. For our analysis, we chose $\alpha$ to correspond to either $2 \sigma$ or $3 \sigma$ (i.e., $\alpha=0.0228$ and $\alpha=0.00135$, respectively). The test statistic $q_{0}$ for each simulated dataset is the profile likelihood ratio, defined as

$$
q_{0}=\left\{\begin{array}{ll}
-2 \ln \left(\frac{\mathcal{L}\left(\vec{\phi}_{\nu_{g}}=0, \hat{\overrightarrow{\hat{\phi}}}_{\nu^{\prime}}\right)}{\mathcal{L}\left(\hat{\bar{\phi}}_{\nu_{k_{g}}} \hat{\bar{\phi}}_{\nu_{k^{\prime}}}\right)}\right), & \hat{\vec{\phi}}_{\nu_{k_{g}}} \geq 0 \\
0, & \hat{\vec{\phi}}_{\nu_{k_{g}}}<0
\end{array},\right.
$$

where the $\phi_{\nu_{k^{\prime}}}$ are treated as nuisance parameters. The hats refer to values that maximize the likelihood, with double hats referring to values maximizing the likelihood subject to the constraint $\phi_{\nu_{k_{g}}}=0$. Note that by definition $q_{0} \geq 0$, with larger values of $q_{0}$ indicating greater incompatibility of the simulated data with the background-only hypothesis $H_{0}$. Thus, we require that the probability $p_{0}$ of having a $q_{0}$ 
value larger (i.e., more incompatible with the data if due to background only) than the "observed" (i.e., than the $q_{0}$ of the simulated dataset) $q_{0}^{\text {obs }}$

$$
p_{0}=\int_{q_{0}^{\mathrm{obs}}}^{\infty} d q_{0} f\left(q_{0} \mid H_{0}\right)
$$

be no larger than $\alpha, p_{0} \leq \alpha$. Here, $f\left(q_{0} \mid H_{0}\right)$ is the PDF of obtaining $q_{0}$ under the background-only hypothesis $H_{0}$. In the large-sample limit, Wilks' theorem ensures that $f\left(q_{0} \mid H_{0}\right)$ is given by $1 / 2$ times a delta function at $q_{0}=0$ plus $1 / 2$ times a $\chi^{2}$ distribution with $n_{g}$ degrees of freedom [67], with $n_{g}$ corresponding to the number of geoneutrino species contributing to the signal. For xenon $n_{g}=1$, for argon $n_{g}=2$, and for germanium and silicon $n_{g}=3$. In the analyses detailed below, we require a value of $\alpha$ corresponding to a $Z \sigma$ significance with $Z=2$ or 3, namely $\alpha(2)=0.0228$ and $\alpha(3)=0.00135$. The value of $\alpha$ corresponding to $Z \sigma$ for $n_{g}=1$ (namely for one degree of freedom) can be obtained by requiring, approximately,

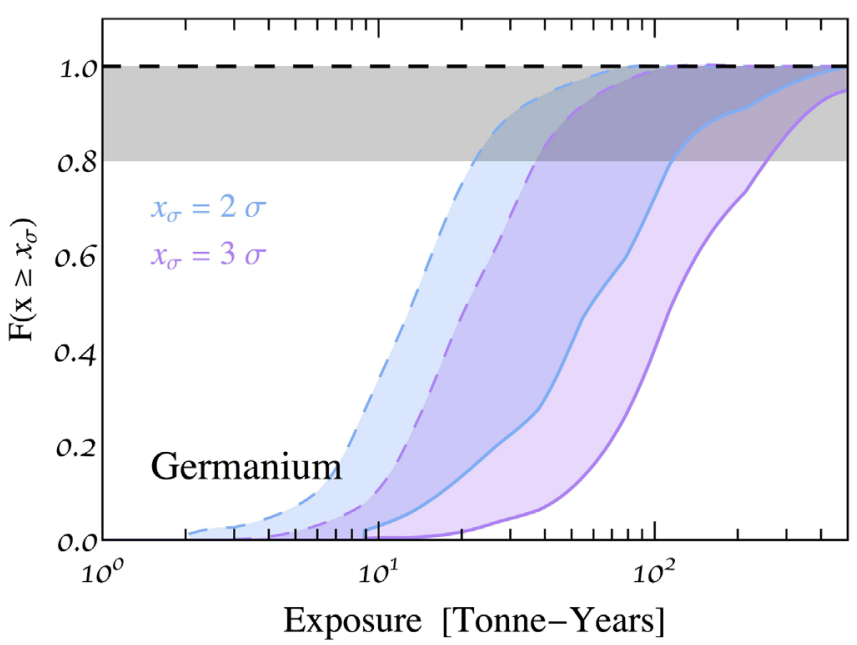

that $q_{0}^{\mathrm{obs}} \geq Z^{2}$. No similar approximate condition exists for $n_{g}=2$ and $n_{g}=2$, and the lower limit of $q_{0}^{\text {obs }}$ is found numerically.

In Fig. 6, we show the fraction $F$ of simulated datasets generated with a particular exposure MT that fulfill this requirement, for $Z=2$ or 3 . Namely, we determine the fraction of simulated datasets that produce $p$ values $p_{0}^{i} \leq$ $\alpha(Z)$ (i.e., those resulting in $\geq Z \sigma$ detection of geoneutrinos) by computing

$$
F\left(\phi_{\nu_{k_{g}}}, \mathrm{MT}\right) \equiv \sum_{i=1}^{N_{\text {sim }}} \frac{1}{N_{\text {sim }}}\left\{\begin{array}{ll}
1 & \text { if } p_{0}^{i} \leq \alpha(Z) \\
0 & \text { if } p_{0}^{i}>\alpha(Z)
\end{array},\right.
$$

where $N_{\text {sim }}$ is the number of simulated datasets with fixed parameters $\left(\phi_{\nu_{k g}}, \mathrm{MT}\right)$.

Notice that the fraction $F$ is the "power" of the test of $H_{0}$ with respect to the alternative hypothesis $H_{\sigma}$, in which $\phi_{\nu_{k_{g}}} \neq 0$ (e.g., Ref. [60], Sec. 40), i.e., $F=(1-\beta)$. This means that the probability of not rejecting $H_{0}$ when the

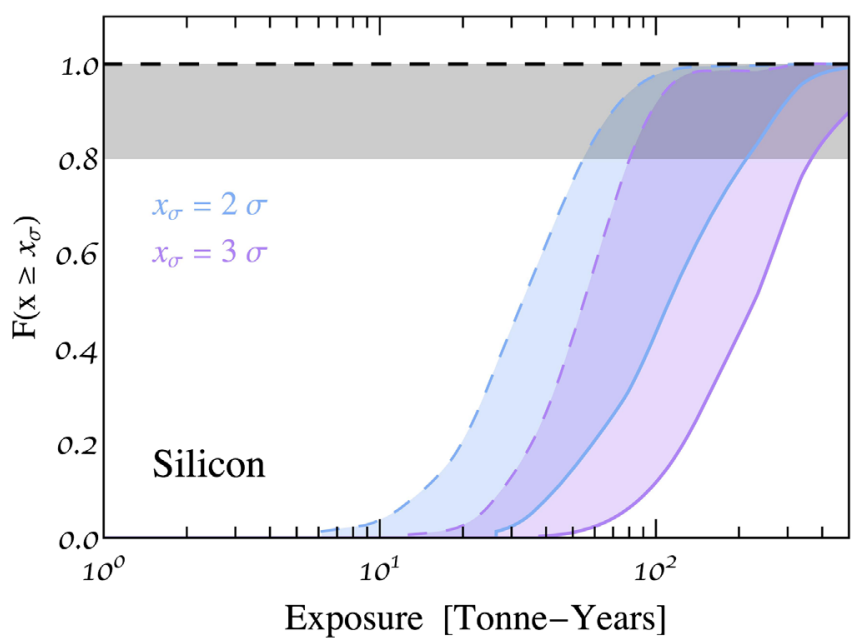

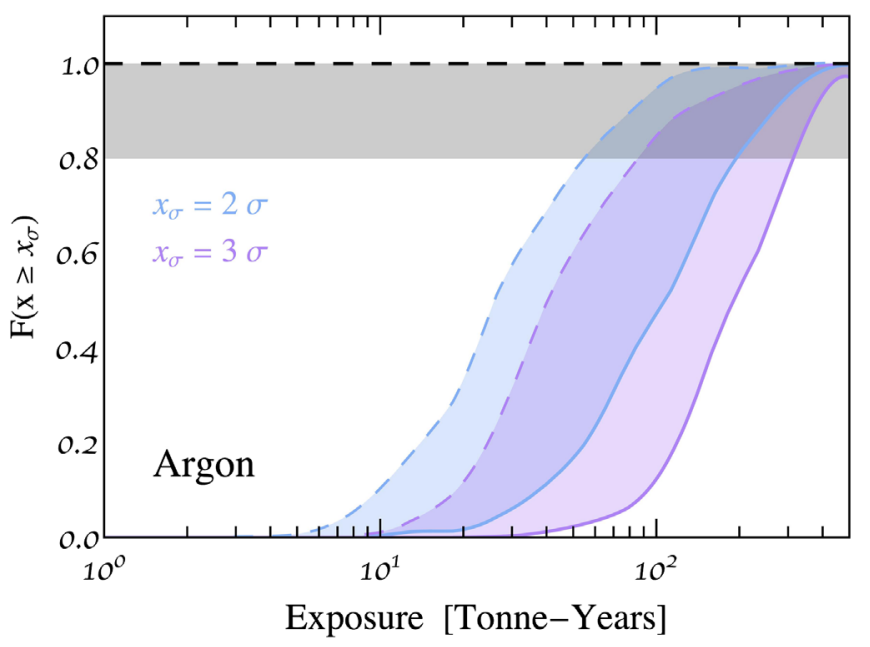

FIG. 6. Minimum exposure needed to obtain a fraction $F$ of simulated datasets in which there is a detection of geoneutrinos at the $2 \sigma$ (blue) and $3 \sigma$ (purple) levels in $\mathrm{Ge}, \mathrm{Si}$, and Ar. The solid (dashed) lines in each band assume the current (and $\times 10^{-2}$ ) systematic errors of Table III. The gray shaded region shows $F>0.8$, where the chance of a type-2 error is small $(<0.2)$. 
"geoneutrino detection" alternative hypothesis $H_{\sigma}$ is true is less than the value $\beta=1-F$.

\section{B. Results}

Figure 6 shows the two bands of fractions $F$ of simulated datasets generated with a particular exposure MT in which there is a detection of geoneutrinos at an $x \sigma$ level with $x \geq Z$, for $Z=2$ (in blue) and $Z=3$ (in purple), as a function of the exposure MT, for Ar, Ge, and Si compositions. The higher exposure boundary (solid line) in each band assumes the systematic errors shown in Table III. The lower exposure boundary (dashed line) of each band assumes instead a very optimistic scenario in which the errors have been divided by a factor of 100 . The gray shaded area highlights the region of $F>0.8$, where the chance of a type- 2 error is small (i.e., $\beta<0.2$ ).

With a Xe target, even a $2 \sigma$ detection of geoneutrinos is not reachable below a 500 ton-year exposure, the largest we consider. The reason is that for the $0.1 \mathrm{keV}$ threshold that we assume, only a small portion of the ${ }^{238} \mathrm{U}$ geoneutrino signal is observable (see Fig. 4).

Figure 6 shows that the most promising detector configuration for geoneutrino detection is based on $\mathrm{Ge}$, because of the lowest potential energy threshold.

\section{SUMMARY}

A measurement of the level of geoneutrino emission is of utmost importance to determining the fraction of the heat emitted by Earth that remains from its formation. The major uncertainty is due to the unknown mantle contribution to the radiogenic heat.

Direct detection experiments have a clear advantage over scintillator-based experiments, which have been used to study geoneutrinos thus far, in that their threshold could be low enough (at least in Ge- and Si-based detectors) to observe geoneutrinos with energies below the kinematic threshold of $1.8 \mathrm{MeV}$, the lowest neutrino energy required for inverse beta decay. This means that ${ }^{40} \mathrm{~K}$ geoneutrinos can also contribute to the signal in direct detection experiments. A measurement of the present level of ${ }^{40} \mathrm{~K}$ with respect to the levels of other geoneutrino types could provide insight into Earth's "volatility curve," a central quantity for Earth's formation history [14].

In this paper we computed the electron and nuclear recoil spectra in different target materials. In Fig. 1, one can see that the geoneutrino electron recoil rate is about 1 order of magnitude lower than the rate of $\mathrm{CNO}$ neutrinos, whose detection sensitivity has been recently explored $[6,10,12]$.

For the detection of nuclear recoils from geoneutrino signal, we considered several potential experimental setups with optimistic thresholds located in the Jinping Underground Laboratory in China, where the expected geoneutrino flux is significantly larger than in other laboratory sites, because of its proximity to the Himalayas, where the crust is the thickest. Assuming that radiogenic heat accounts for most of the heat emitted by our planet, a viable possibility, we study the minimal required exposures for a geoneutrino observation at the $2 \sigma$ and $3 \sigma$ levels, if the background is due only to other neutrinos (solar and reactor neutrinos).

The results of our analysis are shown in Fig. 6. The most promising target material analyzed in this study for geoneutrino detection is germanium, which could have the lowest experimental threshold, if the exposure reaches $\mathcal{O}(10)$ ton-years.

\section{ACKNOWLEDGMENTS}

We thank Carolina Lithgow-Bertelloni, Lars Stixrude, Gerald Schubert, and Linyan Wan for discussions and Sergio Palomares-Ruiz for comments. The work of G. B. G. and V. T. was supported in part by the U.S. Department of Energy (DOE) under Grant No. DE-SC0009937. S. J. W. acknowledges support from Spanish Ministry of Economy and Competitiveness (MINECO) Grants No. FPA201457816-P and No. FPA2017-85985-P, and from European Projects No. H2020-MSCAITN-2015//674896ELUSIVES and No. H2020-MSCA-RISE2015.
[1] J. Billard, L. Strigari, and E. Figueroa-Feliciano, Phys. Rev. D 89, 023524 (2014).

[2] J. Monroe and P. Fisher, Phys. Rev. D 76, 033007 (2007).

[3] G. B. Gelmini, V. Takhistov, and S. J. Witte, J. Cosmol. Astropart. Phys. 07 (2018) 009.

[4] D. Akimov et al. (COHERENT Collaboration), Science 357, 1123 (2017).

[5] M. Pospelov, Phys. Rev. D 84, 085008 (2011).

[6] J. Billard, L. E. Strigari, and E. Figueroa-Feliciano, Phys. Rev. D 91, 095023 (2015).
[7] R. Harnik, J. Kopp, and P. A. N. Machado, J. Cosmol. Astropart. Phys. 07 (2012) 026.

[8] B. Dutta, S. Liao, L. E. Strigari, and J. W. Walker, Phys. Lett. B 773, 242 (2017).

[9] S. Chakraborty, P. Bhattacharjee, and K. Kar, Phys. Rev. D 89, 013011 (2014).

[10] D. G. Cerdeno, J. H. Davis, M. Fairbairn, and A. C. Vincent, J. Cosmol. Astropart. Phys. 04 (2018) 037.

[11] R. Essig, M. Sholapurkar, and T.-T. Yu, Phys. Rev. D 97, 095029 (2018). 
[12] J. L. Newstead, L. E. Strigari, and R. F. Lang, Phys. Rev. D 99, 043006 (2019).

[13] D. McKeen and N. Raj, arXiv:1812.05102.

[14] G. Bellini, A. Ianni, L. Ludhova, F. Mantovani, and W. F. McDonough, Prog. Part. Nucl. Phys. 73, 1 (2013).

[15] D. F. Hollenbach and J. M. Herndon, Proc. Natl. Acad. Sci. U.S.A. 98, 11085 (2001).

[16] T. Araki et al., Nature (London) 436, 499 (2005).

[17] G. Bellini et al. (Borexino Collaboration), Phys. Lett. B 687, 299 (2010).

[18] S. Andringa et al. (SNO+ Collaboration), Adv. High Energy Phys. 2016, 6194250 (2016).

[19] F. An et al. (JUNO Collaboration), J. Phys. G 43, 030401 (2016).

[20] R. Han, Y.-F. Li, L. Zhan, W. F. McDonough, J. Cao, and L. Ludhova, Chin. Phys. C 40, 033003 (2016).

[21] B. Cicenas and N. Solomey (HANOHANO Collaboration), Phys. Procedia 37, 1324 (2012).

[22] J. F. Beacom et al. (Jinping Collaboration), Chin. Phys. C 41, 023002 (2017).

[23] L. Wan, G. Hussain, Z. Wang, and S. Chen, Phys. Rev. D 95 , 053001 (2017).

[24] O. Sramek, B. Roskovec, S. A. Wipperfurth, Y. Xi, and W. F. McDonough, Sci. Rep. 6, 33034 (2016).

[25] M. Leyton, S. Dye, and J. Monroe, Nat. Commun. 8, 15989 (2017).

[26] Z. Wang and S. Chen, arXiv:1709.03743.

[27] P. Cushman et al., in Proceedings Community Summer Study on the Future of U.S. Particle Physics: Snowmass on the Mississippi (CSS2013), Minneapolis, MN, USA, 2013 (2013), arXiv:1310.8327.

[28] E. Aprile et al. (XENON Collaboration), Phys. Rev. Lett. 119, 181301 (2017).

[29] E. Aprile et al. (XENON Collaboration), Phys. Rev. Lett. 121, 111302 (2018).

[30] D. S. Akerib et al. (LUX Collaboration), Phys. Rev. Lett. 118, 021303 (2017).

[31] E. Aprile et al. (XENON Collaboration), Phys. Rev. D 94, 092001 (2016); 95, 059901(E) (2017).

[32] D. S. Akerib et al. (LUX-ZEPLIN Collaboration), arXiv: 1802.06039 .

[33] P. Agnes et al. (DarkSide Collaboration), Phys. Rev. Lett. 121, 081307 (2018).

[34] P. Agnes et al. (DarkSide Collaboration), Phys. Rev. D 98, 102006 (2018).

[35] R. Agnese et al. (SuperCDMS Collaboration), Phys. Rev. D 95, 082002 (2017).

[36] L. Ludhova and S. Zavatarelli, Adv. High Energy Phys. 2013, 425693 (2013).

[37] J. H. Davies and D. R. Davies, Solid Earth 1, 5 (2010).

[38] O. Sramek, W. F. McDonough, E. S. Kite, V. Lekic, S. Dye, and S. Zhong, Earth Planet. Sci. Lett. 361, 356 (2013).

[39] M. Agostini et al. (Borexino Collaboration), Phys. Rev. D 92, 031101 (2015).

[40] Y. Huang, V. Chubakov, F. Mantovani, R. L. Rudnick, and W. F. McDonough, arXiv:1301.0365.

[41] W. C. Haxton, R. G. Hamish Robertson, and A. M. Serenelli, Annu. Rev. Astron. Astrophys. 51, 21 (2013).

[42] N. Grevesse and A. J. Sauval, Space Sci. Rev. 85, 161 (1998).
[43] M. Asplund, N. Grevesse, A. J. Sauval, and P. Scott, Annu. Rev. Astron. Astrophys. 47, 481 (2009).

[44] A. C. Hayes and P. Vogel, Annu. Rev. Nucl. Part. Sci. 66, 219 (2016).

[45] X. B. Ma, W. L. Zhong, L. Z. Wang, Y. X. Chen, and J. Cao, Phys. Rev. C 88, 014605 (2013).

[46] H. Murayama and A. Pierce, Phys. Rev. D 65, 013012 (2001).

[47] M. Baldoncini, I. Callegari, G. Fiorentini, F. Mantovani, B. Ricci, V. Strati, and G. Xhixha, Phys. Rev. D 91, 065002 (2015).

[48] U.S. Energy Information Administration, https://www.eia .gov/todayinenergy/detail.php?id=1490.

[49] V. I. Kopeikin, Yad. Fiz. 75N2, 165 (2012) [Phys. At. Nucl. 75, 143 (2012)].

[50] P. Vogel and J. Engel, Phys. Rev. D 39, 3378 (1989).

[51] T. A. Mueller et al., Phys. Rev. C 83, 054615 (2011).

[52] P. Huber, Phys. Rev. C 84, 024617 (2011); 85, 029901(E) (2012).

[53] D. Adey et al. (Daya Bay Collaboration), arXiv:1808.10836.

[54] P. Vogel, G. K. Schenter, F. M. Mann, and R. E. Schenter, Phys. Rev. C 24, 1543 (1981).

[55] K. Schreckenbach, G. Colvin, W. Gelletly, and F. Von Feilitzsch, Phys. Lett. 160B, 325 (1985).

[56] F. Von Feilitzsch, A. A. Hahn, and K. Schreckenbach, Phys. Lett. 118B, 162 (1982).

[57] A. A. Hahn, K. Schreckenbach, G. Colvin, B. Krusche, W. Gelletly, and F. Von Feilitzsch, Phys. Lett. B 218, 365 (1989).

[58] D. Z. Freedman, D. N. Schramm, and D. L. Tubbs, Annu. Rev. Nucl. Part. Sci. 27, 167 (1977).

[59] R. H. Helm, Phys. Rev. 104, 1466 (1956).

[60] C. Patrignani et al. (Particle Data Group), Chin. Phys. C 40, 100001 (2016).

[61] J.-W. Chen, H.-C. Chi, K.-N. Huang, C. P. Liu, H.-T. Shiao, L. Singh, H. T. Wong, C.-L. Wu, and C.-P. Wu, Phys. Lett. B 731, 159 (2014).

[62] J.-W. Chen, H.-C. Chi, H.-B. Li, C. P. Liu, L. Singh, H. T. Wong, C.-L. Wu, and C.-P. Wu, Phys. Rev. D 90, 011301 (2014).

[63] J.-W. Chen, H.-C. Chi, K.-N. Huang, H.-B. Li, C. P. Liu, L. Singh, H. T. Wong, C.-L. Wu, and C.-P. Wu, Phys. Rev. D 91, 013005 (2015).

[64] J.-W. Chen, H.-C. Chi, C. P. Liu, and C.-P. Wu, Phys. Lett. B 774, 656 (2017).

[65] P. F. de Salas, D. V. Forero, C. A. Ternes, M. Tortola, and J. W. F. Valle, Phys. Lett. B 782, 633 (2018).

[66] M. Agostini et al. (BOREXINO Collaboration), Nature (London) 562, 505 (2018).

[67] G. Cowan, K. Cranmer, E. Gross, and O. Vitells, Eur. Phys. J. C 71, 1554 (2011); 73, 2501(E) (2013).

[68] W. A. Rolke, A. M. Lopez, and J. Conrad, Nucl. Instrum. Methods Phys. Res., Sect. A 551, 493 (2005).

[69] G. Cowan, K. Cranmer, E. Gross, and O. Vitells, arXiv: 1105.3166.

[70] J. Billard, F. Mayet, and D. Santos, Phys. Rev. D 85, 035006 (2012).

[71] E. Aprile et al. (XENON100 Collaboration), Phys. Rev. D 84, 052003 (2011).

[72] F. Ruppin, J. Billard, E. Figueroa-Feliciano, and L. Strigari, Phys. Rev. D 90, 083510 (2014). 